\title{
Mismatch Repair Deficiency Drives Durable Complete Remission by Targeting Programmed Death Receptor 1 in a Metastatic Luminal Breast Cancer Patient
}

\author{
Carlo Fremd $^{\mathrm{a}} \quad$ Mario Hlevnjak $^{\mathrm{b}} \quad$ Marc Zapatka $^{\mathrm{b}} \quad$ Inka Zoernig $^{\mathrm{a}}$ Niels Halama $^{\mathrm{a}}$ \\ Nino Fejzibegovic ${ }^{a}$ Verena Thewes $^{b}$ Peter Lichter $^{b}$ Peter Schirmacher ${ }^{c}$ Matthias Kloor $^{c}$

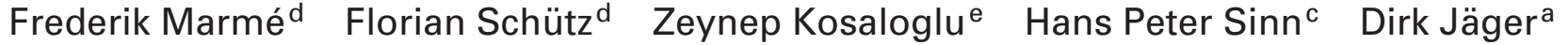 \\ Andreas Schneeweiss ${ }^{a}$
}

a National Center for Tumor Diseases, Department of Medical Oncology, University of Heidelberg, Heidelberg, Germany;

bDivision of Molecular Genetics, German Cancer Research Center, Heidelberg, Germany;

'Department of Applied Tumor Biology, Institute of Pathology, University of Heidelberg, Heidelberg, Germany;

${ }^{\mathrm{d}}$ Department of Obstetrics and Gynecology, University of Heidelberg, Heidelberg, Germany;

e Department of Vaccine Discovery, La Jolla Institute for Allergy and Immunology, La Jolla, CA, USA

\section{Established Facts}

- The blockade of programmed death receptor 1 (PD-1) or its ligand PD-L1 in advanced breast cancer failed in the majority of patients, with modest response rates reported in luminal breast cancer patients.

- As known from colorectal cancer, mismatch repair deficiency is linked to exceptionally high response rates to immune checkpoint blockade.

\section{Novel Insights}

- For the first time, we report on a durable complete remission in a woman with luminal breast cancer after treatment with pembrolizumab, associated with mismatch repair deficiency.

- As a consequence of microsatellite instability, we demonstrated high frequencies of somatic mutations by whole-exome sequencing and identified potential $\mathrm{T}$-cell epitopes by prediction of neoantigens with high affinity binding to one of the patient's HLA.

\section{Keywords}

Programmed death receptor 1, PD-1 - Immune checkpoint blockade - Luminal breast cancer . Pembrolizumab - Mismatch repair deficiency . Mutational load - Microsatellite instability

\section{Abstract}

Background: In the field of breast cancer tumor biology, triple-negative breast cancer patients are the main focus of current clinical trials exploring the use of immune checkpoint inhibitors due to higher frequencies of somatic mutations, neoantigens, and resulting tumor-spe- cific T-cell reactivity. Case Report: Here, we present the case of a 66-year-old woman with metastatic luminal breast cancer that rapidly responded to monotherapy with pembrolizumab, a monoclonal anti-PD-1 antibody. This patient obtained a partial clinical response within the first cycle of treatment and an ongoing durable complete remission after 12 weeks. Except for a transient immune-related thyreoiditis, there were no side effects observed offering remarkable quality of life to the patient. To evaluate the underlying mechanisms, we performed immunohistochemistry, explored the mutational landscape by whole-exome sequencing, and identified

\section{KARGER}

() 2018 S. Karger GmbH, Freiburg

E-Mail karger@karger.com

www.karger.com/brc
Dr. med. Carlo Fremd

National Center for Tumor Diseases

University of Heidelbers

INF 460, 69120 Heidelberg, Germany

carlo.fremd@ med.uni-heidelberg.de 
potential T-cell epitopes by prediction of neoantigens with high affinity binding to one of the patient's HLA. Briefly, we found a strong infiltration of CD8+ T cells without staining for PD-L1 in the tumor stroma. Exome sequencing revealed an enormous frequency of somatic and tumor-specific alterations, mainly $\mathrm{C}>\mathrm{T} / \mathrm{G}>\mathrm{A}$ transitions. The mutational pattern was further linked to genome instability and deficient mismatch repair supported by the loss of MSH6 protein expression and therefore leading to susceptibility to immune checkpoint blockade. Conclusion: Within the overall goal to establish operating procedures for breast cancer immunotherapy, we propose to re-evaluate testing for deficient mismatch repair and to further intensify the search for biomarkers predictive for the success of immune checkpoint modulation including all tumor biologic subtypes of breast cancer.

(c) 2018 S. Karger GmbH, Freiburg

\section{Introduction}

Monoclonal antibodies targeting programmed death receptor 1 (PD-1) and programmed death-ligand 1 (PD-L1) have proven clinical activity in several cancers such as melanoma, lung cancer, and renal cell cancers, and many more trials are underway [1-3]. So far, blockade of PD-1 or PD-L1 in advanced breast cancer failed in the majority of patients, with objective response rates of 5-19\% [4-6]; however, the responses achieved seem to be durable [6]. Best responses have been observed in patients with triple-negative breast cancer (TNBC), who are the main focus of current clinical trials [5]. For luminal type breast cancer, less data is available with limited overall response rates up to $10 \%[7,8]$. This may be explained based on findings that TNBC are generally more immunogenic and therefore more frequently infiltrated with lymphocytic infiltrates as a consequence of more frequent tumor-specific mutations, thus leading to a higher neoantigen load. Moreover, those tumor-infiltrating lymphocytes are linked to a favorable prognosis and predict for response to chemotherapy in early and advanced TNBC and HER2-positive breast cancers [9-11]. As known from numerous clinical trials, upregulation of PD-L1 is associated with higher benefit of therapy and already part of the clinical decision-making in melanoma, lung cancer, and renal cell cancer. In breast cancer patients, a heterogeneous proportion expresses PD-L1 on either tumor or immune cells [12]. However, the mechanistic basis remains inconclusive since many patients with strong staining for PD-L1 do not respond well, while PD-L1negative patients frequently respond. This is supported by inconsistent data for the prognostic value of PD-L1 in breast cancer [13].

In other organ systems, it was shown that tumors with a higher mutational load as a consequence of DNA mismatch repair deficiency leading to microsatellite instability (MSI) turned out to be highly responsive to immune checkpoint blockade [14]. Unlike
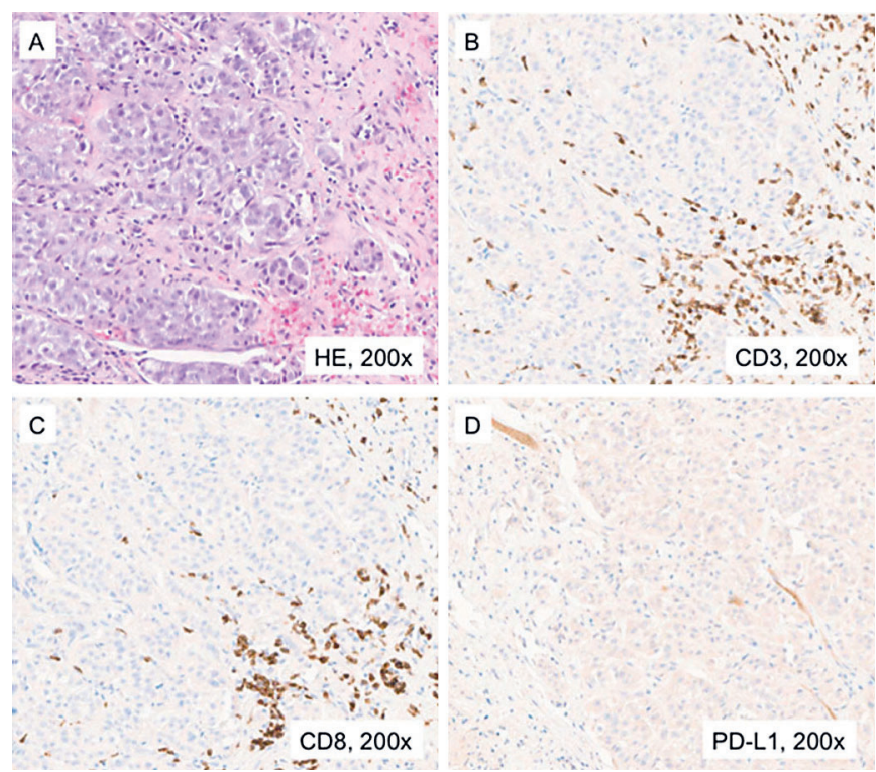

Fig. 1. Immunohistochemistry before the start of anti-PD-1 treatment shows A invasive ductal carcinoma with intermediate differentiation (HE staining) and $\mathbf{B}, \mathbf{C}$ infiltration of CD3+ and CD8+ lymphocytes as well as $\mathbf{D}$ negative staining for PD-L1.

with chromosome instability, MSI-high (MSI-H) tumors reflect a different pathway of carcinogenesis. The 4 genes coding for mismatch repair proteins responsible for DNA repair after recombination or exogenic damage are mutS homologue 2 (MSH2), mutS homologue 6 (MSH6), mutL homologue 1 (MLH1), and postmeiotic segregation increased 2 (PMS2). A defect mismatch repair protein leads to the insertion or deletion of short tandem repeats termed microsatellites and facilitates high frequency somatic mutations. In the case of germline mutations of mismatch repair genes, the resulting hereditary syndrome is known as Lynch syndrome I (colon cancer) or Lynch syndrome II (endometrial, ovarian, and other gastrointestinal cancers), named after Henry Lynch $[15,16]$. In their landmark phase II trial reporting patients with confirmed mismatch repair deficiency (predominantly colorectal cancer patients), Le et al. [17] found $40-78 \%$ response rates to immune checkpoint inhibitors depending on the tumor entity. However, data for breast cancer is heterogenous and implicates a low incidence of mismatch repair deficiency or microsatellite instability [18-25]. Integrating whole-genome sequencing, recent findings report microsatellite instability of less than 2\% [23]. Here, we present the first case of a woman with metastatic, hormone receptor-positive, HER2-negative breast cancer, who achieved a durable complete remission after treatment with pembrolizumab. To the best of our knowledge, this is also the first case of deficient mismatch repair-associated complete remission in breast cancer. Moreover, we discuss implications for future immunotherapy of breast cancer patients with a focus on genomic instability.
Fremd et al. 

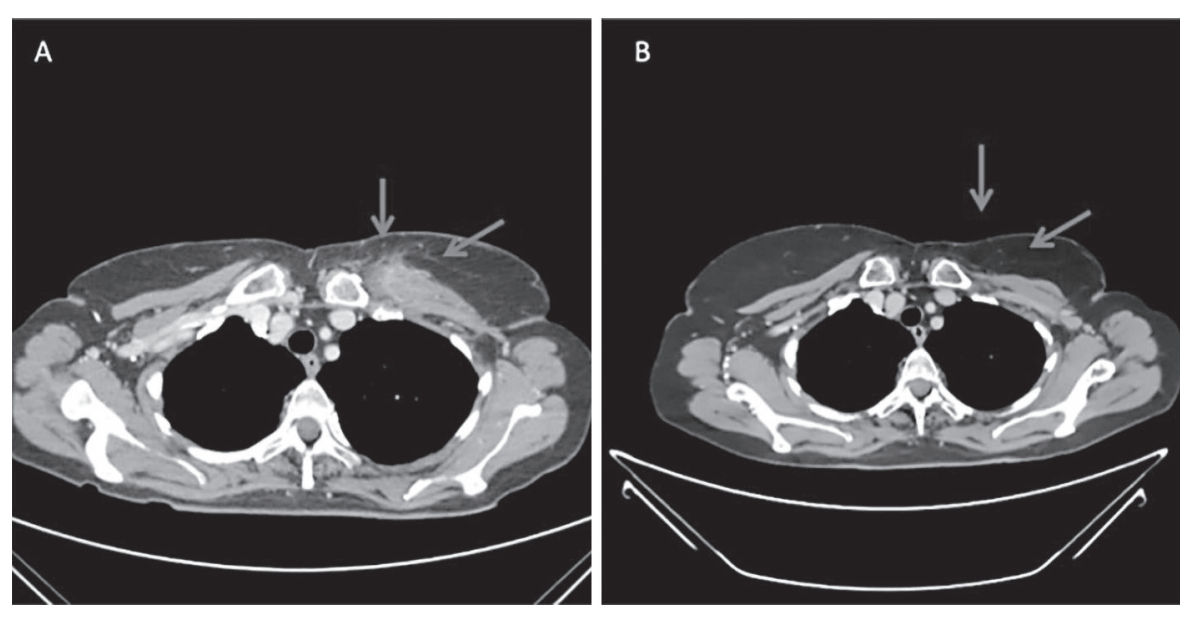

Fig. 2. Computed tomography scans $\mathbf{A}$ before and $\mathbf{B}$ after 6 months of anti-PD-1 treatment demonstrating complete remission of soft tissue metastatic lesions in the left thoracic wall according to immune-related (ir)RECIST criteria.

Fig. 3. Whole-exome sequencing of a pretherapeutic lesion shows $\mathbf{A}$ hypermutation, predominantly $C>T / G>A$ transitions, with overall high numbers of single nucleotide variants $(\mathrm{n}=7,419)$ and $\mathbf{B}$ distribution of mutational signatures in accordance with the catalogue of somatic mutations in cancer (COSMIC).
A

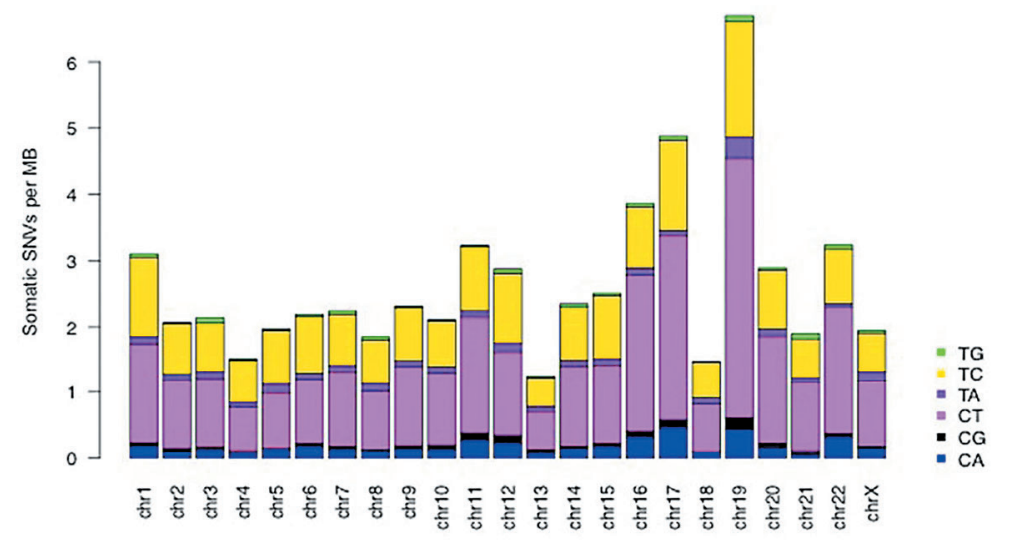

B

\section{Case Report}

A 66-year-old female patient was initially diagnosed in June 2009 with a pT2 $\mathrm{pN} 1$ invasive breast carcinoma of no special type (NST) on the left side. The tumor was found to be estrogen receptor(ER)- and progesterone receptor(PR)positive (ER immunoreactive score 12/12, PR immunoreactive score 3/12). HER2 revealed equivocal expression (2+), but HER2 fluorescent in situ hybridization demonstrated no HER2 copy number gain. Proliferation indices were intermediate (grading 2, Ki-67 15\%). Aiming for curative treatment and with test results being suggestive of an excellent prognosis, the patient underwent breast-conserving surgery and adjuvant chemotherapy with 3 cycles of 5-fluoropyrimidine, epirubicin, and cyclophosphamide (FEC) followed by 3 cycles of docetaxel. Thereafter, primary treatment was completed by radiotherapy of the left breast along with initiation of adjuvant endocrine treatment using an aromatase inhibitor (letrozol) and biannual bisphosponate. After 3 years, ipsilateral axillary lymph node recurrence was diagnosed (ER immunoreactive score 8/12, PR immunoreactive score 5/12). After surgery, adjuvant chemotherapy was performed with capecitabine and vinorelbine followed by radiation of the left axilla. Only 1 month later, the tumor recurred locally in the same area. Due to rapid relapse after first-line endocrine therapy with fulvestrant as a result of secondary endocrine resistance, the treatment was switched to the steroidal aromatase inhibitor exemestane in combination with the mTOR pathway inhibitor everolimus. Combinatorial therapy had to be terminated due to severe mucositis grade 3-4 according to common terminology criteria of adverse events (CTCAE). Proliferation indices, however, did not change during the course of disease and remained within an intermediate stage. In June 2014, the patient was enrolled in a phase I trial evaluating the safety and efficacy of a HER3-directed monoclonal antibody (RO 5479599) along with the application of pertuzumab and docetaxel. However, treatment had to be discontinued due to sustained diarrhea grade 3-4 according to CTCAE. Moreover, imaging studies confirmed progressive disease with involvement of the contralateral thoracic wall in October 2014. Repeated biopsies of the thoracic wall revealed metastasis of ductal invasive carcinoma of the breast. As shown in figure 1, we found a dense infiltration of CD-8-positive T cells without staining for PD-L1 in the metastatic tumor specimens. Further, a weak positive staining signal for PD-1 was assessed, geographically associated with infiltrating lymphocytes (data not shown). When comparing primary tumor with metastatic lesions, no change, neither in PD-1 nor in PD-L1, was observed (data not shown). Nevertheless, immune checkpoint blockade, targeting PD-1 with pembrolizumab $200 \mathrm{mg}$ 

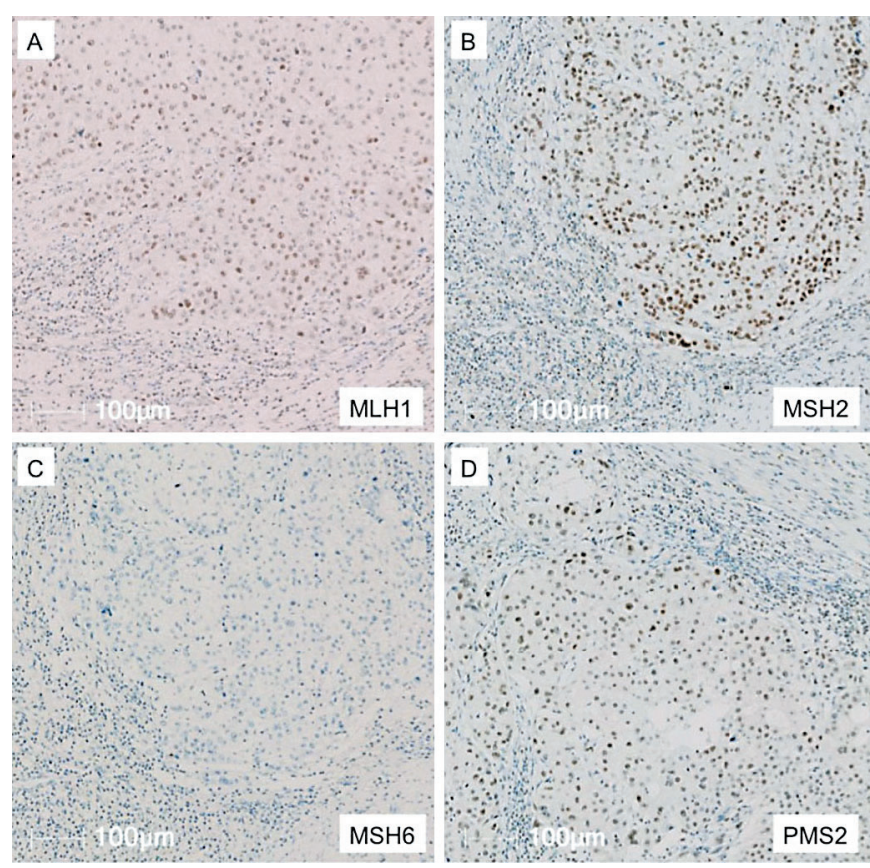

Fig. 4. Immunohistochemical staining of pretherapeutic lesions for deficient mismatch repair proteins reveals positive nuclear staining signals for MLH1, MSH2, PMS2 and loss of MSH6, indicating deficient mismatch repair.

every 3 weeks was started in August 2015. Clinical observations following the first cycle already demonstrated decreasing cutaneous nodules on the left thoracic wall; after the second cycle, a complete clinical remission was obtained. Computed tomography scans, performed every 3 months beginning 2 months after start of pembrolizumab, confirmed an ongoing complete remission without evidence of disease (fig. 2). Except for a transient immune-related thyre oiditis, no side effects were observed offering an excellent quality of life to the patient.

For a deeper understanding, we explored the mutational landscape of one of the metastatic lesions by whole-exome sequencing and found a hypermutated phenotype with an overall high number of 7,419 somatic nucleotide variants (SNV) distributed across all chromosomes (supplementary fig. 1) with a predominance for $\mathrm{C}>\mathrm{T} / \mathrm{G}>\mathrm{A}$ transitions (fig. 3). Of note, a high number of short somatic indels was identified as well as an activating mutation in the ESR1 gene (supplementary table 1). Concerning DNA mismatch repair-associated genes of interest, we identified a somatic frameshift insertion and somatic frameshift deletion in the coding region of $M S H 2$. In contrast, the POLD1 and POLE genes, which have recently been tied to increased mutational load, did not harbor mutations in this case [26]. Along that line, the hypermutated phenotype exhibited high numbers of somatic mutations, and the pattern corresponded to distinct signatures (fig. 3) associated with defective DNA mismatch repair (Signatures 6,15,20,21) as defined by the catalogue of somatic mutations in cancer (COSMIC) [27]. Next, mismatch repair proteins (MLH1, MSH2, MSH6, and PMS2) were assessed by nuclear staining as described in the Methods section. This revealed loss of expression in MSH6 while there were regular nuclear staining signals in the other 3 mismatch repair proteins detected (fig. 4). As a consequence, amplification of 7 microsatellite markers, in accordance with the original Bethesda marker panel, confirmed MSI-H. Importantly, the germline determination of coding genes did not indicate mismatch repair deficiency as a result of Lynch syndrome.

Finally, exceptionally high mutational load was linked to corresponding neoantigens by allele-specific HLA-binding prediction to HLA-I as well as HLA-II. Highly suggestive for an antigen-driven T-cell response and indicated by mutational load as well as the presence of tumor-infiltrating lymphocytes, we identified more than 100 high-affinity binding epitopes (supplementary table 2).

\section{Discussion}

The historic view of breast cancer as an immunologically silent disease has changed. As demonstrated by the prognostic and predictive value of tumor-infiltrating lymphocytes, breast cancer can be detected by the adaptive immune system and arises within a complex interplay of cancer and host cells in the tumor microenvironment. Blocking of PD-1 by a monoclonal antibody (e.g., pembrolizumab, nivolumab) inhibits the negative regulation of $\mathrm{T}$ cells with impressive clinical success in lung cancer, melanoma, and some other solid tumors. However, neoantigens arising from tumor-specific mutations are a prerequisite for the induction of adaptive immunity leading to infiltration of CD8+ T cells and upregulation of effector pathway activity.

Among the breast cancer subtypes, this is mainly attributed to TNBC with higher rates of somatic mutations and tumor-infiltrating lymphocytes [28]. However, all tumor biologic subgroups comprise tumors with high mutational burden [29]. This is demonstrated by the present case, who is, unexpectedly, a hormone receptor-positive luminal B breast cancer patient. However, whole-exome sequencing revealed high numbers of somatic mutations, especially if compared to other ER-positive breast cancers [29]. For the reasons described above and in contrast to TNBC, clinical trials exploring the clinical activity of checkpoint inhibitors in ER-positive breast cancer patients are rare. An expansion of the phase Ib study KEYNOTE 028 enrolled 25 heavily pretreated ER/PR+, HER2-, PD-L1+ patients and reported an overall response rate of $12 \%$, including 3 partial responses. Similarly, our patient was heavily pretreated with several lines of chemotherapy and anti-hormonal treatments, exhibiting secondary endocrine resistance before pembrolizumab was started. Whether distinct therapies in the former history of the patient paved the way for the clinical success of immune checkpoint blockade remains elusive. However, higher response rates have been linked to patients with less previous lines of therapy [30]. Along that line, pembrolizumab as an adjunct to the neoadjuvant treatment of choice in a cohort of early breast cancer patients substantially increased the rate of pathologic complete responses [31]. Another study explored the combination of anti-PDL1 treatment in combination with nab-paclitaxel in metastatic breast cancer demonstrating overall response rates of $41.7-77.8 \%$ in the PD-L1-positive subgroup. Based on these results, the combination of the anti-PD-L1 monoclonal antibody atezolizumab and nabpaclitaxel is under evaluation in frontline treatment, and results are eagerly awaited [30]. Surprisingly, in the tumor specimens of the patient presented here, no staining for PD-L1 was observed in serial immunohistochemical analysis during the course of metastatic disease. Therefore, upregulation of PD-L1 seems not to be the critical immune escape mechanism to overcome immune surveillance. It is important to note that the absence of PD-L1 does not preclude the possibility that immune checkpoint inhibition will lead to disease control as demonstrated in our report. Indeed, even in cancers suggesting a clear predictive value of PD-L1 (e.g., melanoma, lung cancer), clinical and pathologic standards are an ongoing matter of debate [32]. More importantly, not all metastatic lesions could be 
monitored for PD-L1 expression in this case. This represents an open question since site-specific regulation of PD-L1 may have an important impact and remains to be further explored.

In addition to inherited autosomal dominant syndromes, deficiency of DNA mismatch repair proteins (MLH1, MSH2, MSH6, PMS2) as a result of sporadic mutations or promoter methylation (termed microsatellite instable, MSI-H) is the most common cause of increased tumor mutational burden. Here, somatic mismatch repair deficiency was diagnosed by immunohistochemical loss of MSH6 protein expression and corresponding amplification of 7 out of 7 microsatellite markers (MSI-H). In accordance with the literature, not implicating a breast cancer involvement in Lynch syndrome, no germline mutations were found in our case. However, the frequency and impact of other genetic alterations identified in this breast cancer case, especially somatic frameshift insertion and deletion in the coding region of the $\mathrm{MSH} 2$ gene, warrant further research [33]. For the identification of predictive markers, comprehensive genomic and transcriptomic profiling may be of particular interest in large clinical trials investigating immune checkpoint blockade in breast cancer.

From a clinical perspective, Food and Drug Administration approval of pembrolizumab for MSI-H or dMMR cancers, irrespective of the tumor entity, was given in May 2017. 5 uncontrolled, multi-cohort, multi-center, single-arm clinical trials [17, 34] reported on 149 patients with MSI-H or dMMR cancers. In addition to 59 patients diagnosed with colorectal cancer, 14 other cancer entities were included. There were 11 complete responses and 48 partial responses observed with an overall response rate of $39.6 \%$. Since only 2 breast cancer patients were part of these studies, our report is also the first case of $\mathrm{dMMR}$ breast cancer demonstrating a durable complete response to immune checkpoint blockade.

In order to improve patient outcomes in breast cancer, we propose to re-evaluate testing for $\mathrm{dMMR}$ and to further intensify the search for biomarkers predictive for the success of immune checkpoint modulation including all tumor biologic subtypes of breast cancer. With respect to the rapidly increasing number of immunologically active therapies (e.g., mutanome-based vaccines, CAR T cells, other checkpoint inhibitors, immunomodulatory drugs), this is in line with the overall goal to establish operating procedures for the stratification of breast cancer immunotherapy.

\section{Methods}

Serial tissue samples were provided by the NCT Heidelberg Tissue Bank in accordance with its regulations and after approval by the Ethics Committee of Heidelberg University.

\section{Immunohistochemistry and Typing of Microsatellite Instability}

As part of clinical routine, histopathological and tumor biologic parameters were assessed according to protocols of the Department of Pathology at Heidelberg University Hospital by determination of tumor size, nodal involvement, tumor grading, proliferation index (Ki-67), and expression levels of ER, PR, and HER2.
HER2 status was assessed in accordance with current guidelines [35]. ER and PR status was analyzed as percentage of positive nuclear staining. Further, tumor specimens were immunostained for CD3 (clone 2GV6), CD8 (clone C8/144B), CD20 (clone L26), PD-1 (clone NAT105), and PD-L1 (clone E1L3N) first. Second, expression of mismatch repair proteins were analyzed using antibodies for MLH1 (clone M1), MSH6 (clone 44), MSH2 (clone G2191129), and PMS2 (clone EPR3947). Finally, microsatellite instability was determined using the marker panel BAT25, BAT26, D5S346, D2S123, D17S250, complemented by BAT40 and CAT25, as previously described $[36,37]$.

\section{Whole-Exome Sequencing}

Genomic DNA from formalin-fixed and paraffin-embedded tissues was extracted from cell pellets using the QIAGEN DNeasy purification kit (QIAGEN, Hilden, Germany). Libraries of genomic DNA were prepared using the Illumina Paired-End Sample Prep kit (Illumina, Inc., San Diego, SA, USA) according to the manufacturer's guidelines followed by quality control using TapeStation (Agilent, Santa Clara, CA, USA) and fluoroemtric quantification using Qubit (Thermo Fisher Scientific, Waltham, MA, USA). Library preparation for low-input whole-exome sequencing was conducted according to the manufacturer instructions (Agilent Low Input Sure Select v5+UTR) using $200 \mathrm{ng}$ input material. Next, whole-exome sequencing was conducted on the HiSeq 4000 device (Illumina) by pooling 2 samples per lane using 101 bp paired end reads.

\section{Mapping and Analysis of Whole-Exome Sequencing Data}

Exome sequencing reads were aligned to the 1000 Genomes Phase 2 assembly of the Genome Reference Consortium human genome (build 37, version hs37d5) using BWA-MEM [38] (v0.7.8). SNV and short indel calling was performed using in-house pipelines based on SAMtools mpileup (v0.1.19), BCFtools (v0.1.19), and Platypus (v0.8.1) as described previously [39] and updated more recently [40], with the exception of setting the mpileup threshold for minimum read mapping quality in the tumor to 30 .

Supervised mutational signature analysis of high-confidence somatic single nucleotide variants (SNVs) was performed using nonnegative matrix factorization formalism as described previously [41]. A predefined set of 30 canonical mutational signatures was used for decomposition of somatic SNVs (cancer.sanger.ac.uk/cosmic/signatures), which were further re-normalized using the observed trinucleotide frequencies in the human exome to the ones of the human genome.

\section{Neoantigen Prediction}

First, the HLA type of the patient was determined using the HLA typing tool Phlat to infer the 4-digit HLA genotype from the corresponding whole exome data. Second, NetMHCpan (version 2.8) and NetMHCIIpan (version 3.0) were used to predict allele-specific HLA-binding for both, HLA class I and HLA class II. To annotate 
DNA mutations to protein sequences, the stand-alone software packages of the tools were integrated in a shell-based analysis pipeline and HLA-binding predictions performed for the mutated and corresponding wildtype protein stretches. Neoepitopes were predicted by defining all 9-11mers resulting from mutations and determining whether the predicted binding affinity to one of the patient's HLA alleles was $<500 \mathrm{nM}$, dismissing those with a lower binding affinity. In order to remove neoantigens arising from genes without expression, predicted neoantigens were compared with TCGA RNAseq data for ductal invasive breast carcinoma.

\section{Online Supplemental Material}

Suppl. table 1. Identified somatic mutations by whole-exome sequencing

Suppl. table 2. Predicted neoantigens with high-affinity binding

Suppl. fig. 1. Intermutation distances of somatic mutations.

To access the supplemental material please refer to www.karger. com/?DOI=492580.

\section{Patient Consent and Ethical Statement}

The patient provided signed written consent for sample and gene analysis according to the German Gene Diagnosis Act.

\section{Acknowledgement}

We gratefully acknowledge the patient guidance and care provided by Andrea Kohl and the medical staff of the gynecological oncology unit at NCT Heidelberg. We also thank the members of the High Throughput Sequencing unit of the Genomics \& Proteomics Core Facility, DKFZ, for providing excellent whole-exome sequencing services.

This project was supported by the Heidelberg Center for Personalized Oncology Program (DKFZ-HIPO).

\section{Author's Contributions}

C. Fremd and A. Schneeweiß designed the treatment and research plan, collected and interpreted the data, and wrote the manuscript. M. Hlevnjak and M. Zapatka analyzed and interpreted exome sequencing data and reviewed the manuscript. H.P. Sinn and M. Kloor carried out immunohistochemistry and microsatellite typing. V. Thewes, P. Lichter, D. Jäger, I. Zörnig, N. Halama, Z. Kosaloglu, F. Schütz, F. Marmé, P. Schirmacher, and N. Fejzibegovic contributed to interpretation of data. All authors read and approved the final version.

\section{Disclosure Statement}

The authors declare no potential conflicts of interest.

\section{References}

1 Melero I, Berman DM, Aznar MA, et al: Evolving synergistic combinations of targeted immunotherapies to combat cancer. Nat Rev Cancer 2015;15:457-472.

2 Topalian SL, Hodi FS, Brahmer JR, et al: Safety, activity, and immune correlates of anti-PD-1 antibody in cancer. N Engl J Med 2012;366:2443-2454.

3 Postow MA, Callahan MK, Wolchok JD: Immune checkpoint blockade in cancer therapy. J Clin Oncol 2015;33:1974-1982.

4 Emens LA, Braiteh FS, Cassier P, et al: Abstract PD1-6: Inhibition of PD-L1 by MPDL3280A leads to clinical activity in patients with metastatic triple-negative breast cancer. Cancer Res 2015;75(suppl):abstr PD1-6.

5 Nanda R, Chow LQ, Dees EC, et al: Pembrolizumab in patients with advanced triple-negative breast cancer: phase Ib KEYNOTE-012 study. J Clin Oncol 2016;34: 2460-2467.

6 Emens LA, Kok M, Ojalvo LS: Targeting the programmed cell death-1 pathway in breast and ovarian cancer. Curr Opin Obstet Gynecol 2016;28:142-147.

7 Rugo H, Delord J-P, Im S-A, et al: Abstract S507:Preliminary efficacy and safety of pembrolizumab (MK-3475) in patients with PD-L1-positive, estrogen receptor-positive (ER+)/HER2-negative advanced breast cancer enrolled in KEYNOTE-028. Cancer Res 2016;76(suppl):abstr S5-07.

8 Dirix L, Takacs I, Nikolinakos P, et al: Abstract S1-04: Avelumab (MSB0010718C), an anti-PD-L1 antibody, in patients with locally advanced or metastatic breast cancer: a phase Ib JAVELIN solid tumor trial. Cancer Res 2016;76(suppl):S1-04.
9 Luen SJ, Salgado R, Fox S, et al: Tumour-infiltrating lymphocytes in advanced HER2-positive breast cancer treated with pertuzumab or placebo in addition to trastuzumab and docetaxel: a retrospective analysis of the CLEOPATRA study. Lancet Oncol 2017;18:52-62.

10 Loi S, Michiels S, Salgado R, et al: Tumor infiltrating lymphocytes are prognostic in triple negative breast cancer and predictive for trastuzumab benefit in early breast cancer: results from the FinHER trial. Ann Oncol 2014;25:1544-1550.

11 Denkert C, von Minckwitz G, Brase JC, et al: Tumorinfiltrating lymphocytes and response to neoadjuvant chemotherapy with or without carboplatin in human epidermal growth factor receptor 2-positive and triplenegative primary breast cancers. J Clin Oncol 2015;33: 983-991.

12 Ali HR, Glont SE, Blows FM, et al: PD-L1 protein expression in breast cancer is rare, enriched in basal-like tumours and associated with infiltrating lymphocytes. Ann Oncol 2015;26:1488-1493.

13 Sabatier R, Finetti P, Mamessier E, et al: Prognostic and predictive value of PDL1 expression in breast cancer. Oncotarget 2015;6:5449-5464.

14 Chan TA, Wolchok JD, Snyder A: Genetic basis for clinical response to CTLA-4 blockade in melanoma. N Engl J Med 2015;373:1984.

15 Jasperson KW, Tuohy TM, Neklason DW, Burt RW: Hereditary and familial colon cancer. Gastroenterology 2010;138:2044-2058.

16 Umar A, Boland CR, Terdiman JP, et al: Revised Bethesda Guidelines for hereditary nonpolyposis colorectal cancer (Lynch syndrome) and microsatellite instability. J Natl Cancer Inst 2004;96:261-268.

17 Le DT, Uram JN, Wang H, et al: PD-1 blockade in tumors with mismatch-repair deficiency. N Engl J Med 2015;372:2509-2520.
18 Shaw JA, Walsh T, Chappell SA, et al: Microsatellite instability in early sporadic breast cancer. Br J Cancer 1996;73:1393-1397.

19 Paulson TG, Wright FA, Parker BA, Russack V, Wahl GM: Microsatellite instability correlates with reduced survival and poor disease prognosis in breast cancer. Cancer Res 1996;56:4021-4026.

20 Anbazhagan R, Fujii H, Gabrielson E: Microsatellite instability is uncommon in breast cancer. Clin Cancer Res 1999;5:839-844.

21 Adem C, Soderberg CL, Cunningham JM, et al: Microsatellite instability in hereditary and sporadic breast cancers. Int J Cancer 2003; 107:580-582.

22 Kuligina E, Grigoriev MY, Suspitsin EN, et al: Microsatellite instability analysis of bilateral breast tumors suggests treatment-related origin of some contralateral malignancies. J Cancer Res Clin Oncol 2007;133:5764.

23 Davies H, Morganella S, Purdie CA, et al: Whole-genome sequencing reveals breast cancers with mismatch repair deficiency. Cancer Res 2017;77:4755-4762.

24 Wen YH, Brogi E, Zeng Z, et al: DNA mismatch repair deficiency in breast carcinoma: a pilot study of triplenegative and non-triple-negative tumors. Am J Surg Pathol 2012;36:1700-1708.

25 Mills AM, Dill EA, Moskaluk CA, et al: The relationship between mismatch repair deficiency and PD-L1 expression in breast carcinoma. Am J Surg Pathol 2018;42:183-191.

26 Jansen AM, van Wezel T, van den Akker BE, et al: Combined mismatch repair and POLE/POLD1 defects explain unresolved suspected Lynch syndrome cancers. Eur J Hum Genet 2016;24:1089-1092. 
27 Alexandrov LB, Stratton MR: Mutational signatures: the patterns of somatic mutations hidden in cancer genomes. Curr Opin Genet Dev 2014;24:52-60.

28 Loi S, Adams S, Schmid P, et al: LBA13 - Relationship between tumor infiltrating lymphocyte (TIL) levels and response to pembrolizumab (pembro) in metastatic triple-negative breast cancer (mTNBC): results from KEYNOTE-086. Ann Oncol 2017;28(suppl 5):v605-v649.

29 Budczies J, Bockmayr M, Denkert C, et al: Classical pathology and mutational load of breast cancer - integration of two worlds. J Pathol Clin Res 2015;1:225-238.

30 Adams S, Diamond JR, Hamilton EP, et al: Phase Ib trial of atezolizumab in combination with nab-paclitaxel in patients with metastatic triple-negative breast cancer (mTNBC). J Clin Oncol 2016;34(suppl):1009.

31 Nanda R, Liu MC, Yau C, et al: Pembrolizumab plus standard neoadjuvant therapy for high-risk breast cancer (BC): results from I-SPY 2. J Clin Oncol 2017; 35(suppl):506.
32 Daud AI, Wolchok JD, Robert C, et al: Programmed death-ligand 1 expression and response to the antiprogrammed death 1 antibody pembrolizumab in melanoma. J Clin Oncol 2016;34:4102-4109.

33 Schwitalle Y, Kloor M, Eiermann S, et al: Immune response against frameshift-induced neopeptides in HNPCC patients and healthy HNPCC mutation carriers. Gastroenterology 2008;134:988-997.

34 Le DT, Durham JN, Smith KN, et al: Mismatch repair deficiency predicts response of solid tumors to PD-1 blockade. Science 2017;357:409-413.

35 Wolff AC, Hammond ME, Hicks DG, et al: Recom mendations for human epidermal growth factor receptor 2 testing in breast cancer: American Society of Clinical Oncology/College of American Pathologists clinical practice guideline update. Arch Pathol Lab Med 2014;138:241-256.

36 Boland CR, Thibodeau SN, Hamilton SR, et al: A National Cancer Institute Workshop on Microsatellite Instability for cancer detection and familial predisposition: development of international criteria for the determination of microsatellite instability in colorectal cancer. Cancer Res 1998;58:5248-5257.
37 Findeisen P, Kloor M, Merx S, et al: T25 repeat in the 3' untranslated region of the CASP2 gene: a sensitive and specific marker for microsatellite instability in colorectal cancer. Cancer Res 2005;65:8072-8078.

$38 \mathrm{Li} \mathrm{H}$ : Aligning sequence reads, clone sequences and assembly contigs with BWA-MEM. Cornell University Library, arXiv:1303.3997v2, 2013.

39 Jones DT, Jäger N, Kool M, et al: Dissecting the genomic complexity underlying medulloblastoma. $\mathrm{Na}$ ture 2012;488:100-105.

40 Chudasama P, Mughal SS, Sanders MA, et al: Integrative genomic and transcriptomic analysis of leiomyosarcoma. Nat Commun 2018;9:144.

41 Alexandrov LB, Nik-Zainal S, Wedge DC, Campbell PJ, Stratton MR: Deciphering signatures of mutational processes operative in human cancer. Cell Rep 2013;3: 246-259. 